\section{¿Cuánto investigan los estudiantes de medicina en pregrado?}

\section{How many undergraduate medical students are involved in medical research?}

\section{Sr. Editor:}

El desarrollo de nuevo conocimiento es esencial para el progreso de la medicina. Una carta al editor publicada en esta revista, mostró preocupación por el estado actual de la investigación de los estudiantes de pregrado ${ }^{1}$. Se comentó una disminución catastrófica del número de trabajos presentados en el Congreso Científico Nacional de Estudiantes de Medicina (CCNEM) entre los años 2005 y 2013. A partir de esos datos se podría concluir que los estudiantes de medicina están investigando menos. Sin embargo, consideramos que dicha afirmación puede ser limitada y no atenerse a la realidad. Muchos factores influyen en que los alumnos de pregrado presenten o no sus trabajos en el CCNEM: desconocimiento de su existencia, presentación en otros congresos nacionales o internacionales, falta de interés, entre otras ${ }^{2,3} \cdot$ ¿Significará realmente que investigan menos?

En Chile no hay estudios publicados que evalúen la participación de los estudiantes de pregrado en investigación biomédica. Por tanto, nos parece necesario complementar con algunos datos obtenidos por nosotros: revisamos retrospectivamente los artículos publicados online en la Revista Médica de Chile durante los años 2006 a 2010. Se consideraron "artículos de investigación”, "casos clínicos", "artículos de revisión” y "otros artículos" (incluyendo artículos especiales, ética-médica, medicina basada en evidencia, educación médica y salud pública). Por cada artículo registramos el número de autores, número de autores correspondientes a estudiantes de medicina y número de estudiantes de medicina como primer autor. Se identificaron 1.014 artículos (539 artículos de investigación, 209 casos clínicos, 121 artículos de revisión y 135 catalogados como “otros artículos"). En el 14,1\% del total de artículos participaron estudiantes de medicina. Sólo $2,3 \%$ figuraron como primer autor. Del total de 5.140 autores, 257 eran estudiantes de medicina (5\%). No hubo diferencias estadísticamente significativas por grupo de artículos.
Considerando la baja participación de los estudiantes de medicina en una de las principales revistas médicas de nuestro país, urge evaluar las causas del hecho y potenciales soluciones.

Otro tema muy interesante que comentaron Clouet-Huerta et $\mathrm{al}^{1}$, es que los alumnos de pregrado de la Universidad de Chile y la Pontificia Universidad Católica de Chile no destacaron en el número de investigaciones presentadas en el CCNEM. Esto pese a que los datos de CONICYT sitúan a estos planteles como los dos principales centros de educación superior de investigación en nuestro país, tanto en número de publicaciones como en recursos invertidos. Respecto a este punto, queremos agregar algunos comentarios: Primero, es importante considerar que el número no necesariamente toma relación con el tiempo dedicado por el estudiante o con la calidad del trabajo; segundo, es necesario obtener información de la participación de los alumnos en el resto de los múltiples congresos existentes en la actualidad, tanto de carácter nacional como internacional. Además, queremos resaltar el concepto "investigaciones publicadas". Tanto que se presenten menos trabajos en el CCNEM, como que no se publiquen, no significa necesariamente que los alumnos no investiguen. Sin embargo, a partir de los datos expuestos, es presumible que la participación en investigación biomédica por parte de los estudiantes de pregrado sea indeseablemente baja.

Es un hecho la baja contribución de los estudiantes de medicina en los artículos publicados en la Revista Médica de Chile. Realizar modificaciones a nivel nacional en las mallas curriculares de la carrera de medicina, que incluyan investigación formal, podría contribuir a mejorar esta situación. Recientemente, un grupo canadiense publicó una revisión extensa de carácter cualitativo, evidenciando que la obligatoriedad de investigar en pregrado no disminuye la calidad de la investigación ni la productividad del estudiante. Asimismo, recomiendan crear un programa con expectativas claras y razonables para los alumnos, facilitar el contacto con los tutores y la Facultad, proveer el suficiente tiempo para que los estudiantes puedan dedicarse a su investigación y generar mecanismos por los cuales ellos sean reconocidos por su participación ${ }^{4}$.

Tenemos la convicción de que la formación de médicos para nuestro país, en un ambiente de investigación novedosa y constante, los hará 
ser más críticos en el ejercicio de la medicina en beneficio del paciente.

\author{
Esteban Araos-Baeriswyl ${ }^{l, a}$, \\ Catherina Moll-Manzur ${ }^{b}$ \\ ${ }^{1}$ Departamento de Medicina Interna, Facultad \\ de Medicina, Pontificia Universidad Católica de \\ Chile. Santiago, Chile. \\ ${ }^{a}$ Residente. \\ ${ }^{b}$ Interna de Medicina, Escuela de Medicina, \\ Pontificia Universidad Católica de Chile.
}

\title{
Referencias
}

1. Clouet-Huerta D, Correa K. Investigación médica en pregrado: ¿qué está sucediendo en Chile? Rev Med Chile 2014; 142 (11): 1488-90.

2. Karani R, Ognibene FP, Fallar R, Gliatto P. Medical

\section{¿Cuánto investigan los estudiantes de medicina en pregrado? Réplica}

\section{How many undergraduate medical students are involved in medical research? A reply}

\section{Sr. Editor:}

Aludiendo a la incógnita planteada por Araos-Baeriswyl et al. en relación al estudio presentado previamente: “ ¿isignificará realmente que investigan menos?” concordamos que en Chile existen escasos estudios que permitan dilucidar y profundizar al respecto, haciendo necesarios trabajos que abarquen de forma íntegra la temática. Sin embargo, creemos que el número de trabajos presentados en Congresos de Estudiantes de Medicina puede reflejar bien este tópico, considerando que este evento científico nacional es organizado y avalado por Sociedades Científicas de Estudiantes de Medicina de 16 de 18 Facultades de Medicina respaldadas por la Asociación de Facultades de Medicina de Chile, exceptuándose la Pontificia Universidad Católica de Chile y la Universidad Finis Terrae ${ }^{1,2}$.

Por otro lado, es importante destacar de los datos presentados por Araos-Baeriswyl et al. que sólo 23 (2,3\%) trabajos de un total de 1.014 publicaciones que incorporaban estudiantes de students' experiences with authorship in biomedical research: a national survey. Acad Med 2013; 88 (3): 364-8.

3. Ávila $M$, Rodríguez-Restrepo A. The importance of research in undergraduate medical education. Medwave 2014; 14 (10): e6032.

4. Chang Y, Ramnanan C. A Review of Literature on Medical Students and Scholarly Research: Experiences, Attitudes, and Outcomes. Acad Med 2015; Apr 4. [Epub ahead of print].

Financiamiento: ninguno.

Conflicto de intereses: ninguno por declarar.

Correspondencia a:

Dr. Esteban Araos-Baeriswyl

Departamento de Medicina Interna, Pontificia Universidad Católica de Chile.

Lira 63, Santiago, Santiago de Chile.

earaos@med.puc.cl

medicina en la Revista Médica de Chile, durante el período 2006-2010, tenían a un estudiante de medicina como primer autor. Este dato resalta, no sólo porque avala nuestro punto sobre el bajo aporte estudiantil en las ciencias, sino ya que se ha observado que en Latinoamérica los estudiantes de medicina tienden a desarrollar trabajos de investigación originales ${ }^{3}$, ideas y datos nuevos que no han sido publicados y que no son de conocimiento público. Si bien tratamos con datos empíricos faltando antecedentes para avalarlo, cabe hacerse la pregunta: ¿qué ocurre con todas estas investigaciones originales en Chile? ¿Se estarán perdiendo?

Respecto a las consideraciones que se mencionaron sobre la validez de suponer como definitiva la idea de que la menor cantidad de estudios presentados en congresos implica que los estudiantes no investigan, concordamos en que es imposible obtener una conclusión total desde una visión parcial; sin embargo, da luces de lo que pudiese estar ocurriendo a nivel nacional.

Según un estudio en el Reino Unido sólo el 8\% de los estudiantes de medicina en el país no estaba interesados en investigar, pero el 55\% del total no había tenido la oportunidad de hacerlo, situación que pudiese estar reflejándose también en nuestro país y es, tal vez, la razón de la disminución de trabajos presentados. 
Por otra parte, no sólo importa el número, sino también la calidad, la que se corrobora mediante la publicación científica. De otro modo, no se estaría cumpliendo con el objetivo final de la investigación $^{4}$, es decir, la contribución y socialización del conocimiento. Situaciones particularmente preocupantes, como ha sido señalado en otras investigaciones en donde sólo 125 (31,9\%) de un total de 392 resúmenes presentados en Congresos Chilenos de Gastroenterología durante el período 1998-2002 están publicados o en vías de hacerlo ${ }^{5}$, o en Perú, donde sólo se publicaron $52(9,8 \%)$ de 532 resúmenes totales presentados en Congresos Científicos de Estudiantes de Medicina del Perú durante el período 2002-20096.

Finalmente, concordamos en que es necesario realizar cambios profundos en las mallas curriculares de las distintas Facultades de Medicina nacionales, no sólo con el fin de potenciar esta área pobremente desarrollada en el país y que tiene enormes implicancias económicas para el Estado, sino para desarrollar una visión del país pro investigación biomédica. De esta manera no sólo se formarán estudiantes de medicina cada vez más íntegros, al ir adquiriendo habilidades que se desarrollan con la investigación científica, como la lectura crítica, mejor resolución de casos clínicos, mejora en la comunicación, entre otros $^{7}$, sino que también generen una verdadera contribución al desarrollo del país como entes de cambio y progreso.

Diego E. Clouet-Huerta ${ }^{1, a}$, Katherine Correa U., ${ }^{2, b}$ ${ }^{1}$ Escuela de Medicina, Facultad de Medicina. Universidad Austral de Chile. Valdivia, Chile. ${ }^{2}$ Escuela de Antropología, Facultad de Filosofía y Humanidades. Universidad Austral de Chile. Valdivia, Chile. ${ }^{a}$ Estudiante de Medicina. ${ }^{b}$ Estudiante de Antropología.

\section{Referencias}

1. Asociación Nacional Científica de Estudiantes de Medicina de Chile [Internet]. Sociedades Miembro. Santiago: Asociación Nacional Científica de Estudiantes de Medicina de Chile; c2014 [Citado el 21 de julio de 2015]. Disponible en: www.anacem.cl/sociedades-miembro/

2. Ministerio de Salud [Internet]. Facultades de Medicina. Santiago: Ministerio de Salud; 2015 [Citado el 21 de julio de 2015]. Disponible en: www.web.minsal.cl/ facultades_de_medicina.

3. Cabrera-Samith I, Oróstegui-Pinilla D, Ángulo-Bazán Y, Mayta-Tristán P, Rodríguez-Morales A. Revistas científicas de estudiantes de medicina en Latinoamérica. Rev Med Chile 2010; 138 (11): 1451-5.

4. Clouet D. Publicar nuestra investigación: ¿Qué nos detiene? (carta). Rev Med Chile 2013; 141 (12): 1605-6.

5. Weitz J, Silva H. Presentación versus publicación: análisis de trabajos presentados en Congresos Chilenos de Gastroenterología 1998 al 2002. Gastroenterol Latinoam 2005; 16 (3): 287-92.

6. Toro L, Pereyra R, Nizama A, Fernando L, Vélez E, Galán E, et al. Publicación de los trabajos presentados a los congresos científicos de estudiantes de medicina, Perú 2002-2009: Características y factores asociados. Rev Perú Med Exp. Salud Pública 2012; 29 (4): 461-8.

7. Griffin MF, Hindocha S. Publication practices of medical student at British medical schools: Experience, attitudes and barriers to publish. Med Teach 2011; 33 (1): e1-9.
Correspondencia a:

Diego E. Clouet Huerta.

Italia \#1740. Valdivia, Chile.

dclouet@hotmail.com 